\title{
Sirtuin 6 regulates glucose-stimulated insulin secretion in mouse pancreatic beta cells
}

\author{
Xiwen Xiong ${ }^{1}$ - Gaihong Wang ${ }^{1} \cdot$ Rongya Tao $^{1} \cdot$ Pengfei $\mathrm{Wu}^{2}$ • Tatsuyoshi Kono ${ }^{3}$. \\ Kevin $\mathrm{Li}^{4} \cdot$ Wen-Xing Ding ${ }^{4}$ Xin Tong ${ }^{3,5}$ - Sarah A. Tersey ${ }^{6}$ - Robert A. Harris ${ }^{1,2}$. \\ Raghavendra G. Mirmira $^{6}$ - Carmella Evans-Molina ${ }^{2,3,5} \cdot$ X. Charlie Dong ${ }^{1}$
}

Received: 23 April 2015 / Accepted: 22 September 2015 / Published online: 15 October 2015

(C) Springer-Verlag Berlin Heidelberg 2015

\begin{abstract}
Aims/hypothesis Sirtuin 6 (SIRT6) has been implicated in ageing, DNA repair and metabolism; however, its function in pancreatic beta cells is unclear. The aim of this study is to elucidate the role of SIRT6 in pancreatic beta cells.

Methods To investigate the function of SIRT6 in pancreatic beta cells, we performed Sirt6 gene knockdown in MIN6 cells and generated pancreatic- and beta cell-specific Sirt6 knockout mice. Islet morphology and glucose-stimulated insulin secretion (GSIS) were analysed. Glycolysis and oxygen consumption rates in SIRT6-deficient beta cells were measured. Cytosolic calcium was monitored using the Fura-2-AM fluorescent probe (Invitrogen, Grand Island, NY, USA). Mitochondria were analysed by immunoblots and electron microscopy.

Results Sirt6 knockdown in MIN6 beta cells led to a significant decrease in GSIS. Pancreatic beta cell Sirt6 knockout
\end{abstract}

X. Charlie Dong

xcdong@iu.edu

1 Department of Biochemistry and Molecular Biology, Indiana University School of Medicine, 635 Barnhill Drive, MS1021D, Indianapolis, IN 46202, USA

2 Richard Roudebush Veterans Affairs Medical Center, Indianapolis, IN, USA

3 Department of Medicine, Indiana University School of Medicine, Indianapolis, IN, USA

4 Department of Pharmacology, Toxicology and Therapeutics, University of Kansas Medical Center, Kansas City, KS, USA

5 Department of Cellular and Integrative Physiology, Indiana University School of Medicine, Indianapolis, IN, USA

6 Department of Pediatrics, Indiana University School of Medicine, Indianapolis, IN, USA mice showed a $\sim 50 \%$ decrease in GSIS. The knockout mouse islets had lower ATP levels compared with the wild-type controls. Mitochondrial oxygen consumption rates were significantly decreased in the SIRT6-deficient beta cells. Cytosolic calcium dynamics in response to glucose or potassium chloride were attenuated in the Sirt6 knockout islets. Numbers of damaged mitochondria were increased and mitochondrial complex levels were decreased in the SIRT6-deficient islets. Conclusions/interpretation These data suggest that SIRT6 is important for GSIS from pancreatic beta cells and activation of SIRT6 may be useful to improve insulin secretion in diabetes.

Keywords Beta cell · Calcium · Glucose metabolism · Insulin secretion $\cdot$ Mitochondria $\cdot$ SIRT6

$\begin{array}{ll}\text { Abbreviations } \\ \text { ADPR } & \text { ADP ribose } \\ \text { bMko } & \text { Sirt6 beta cell-specific knockout } \\ \text { bPko } & \text { Sirt6 pancreas-specific knockout } \\ {\left[\mathrm{Ca}^{2+}\right]_{\mathrm{i}}} & \text { Intracellular calcium } \\ \text { ECAR } & \text { Extracellular acidification rate } \\ \text { ES } & \text { Embryonic stem } \\ \text { GSIS } & \text { Glucose-stimulated insulin secretion } \\ \text { H3K9 } & \text { Histone H3 at lysine } 9 \\ \text { HFD } & \text { High-fat diet } \\ \text { ITT } & \text { Insulin tolerance test } \\ \text { KCl } & \text { Potassium chloride } \\ \text { KIC } & \alpha \text {-Ketoisocaproate } \\ \text { NIDDK } & \text { National Institute of Diabetes and Digestive } \\ & \text { and Kidney Diseases } \\ \text { NIH } & \text { National Institutes of Health } \\ \text { OAADPR } & O \text {-Acetyl-ADP ribose } \\ \text { OCR } & \text { Oxygen consumption rate }\end{array}$

Abbreviations

bMko Sirt6 beta cell-specific knockout

Sirt6 pancreas-specific knockout

Intracellular calcium

Extracellular acidification rate

Embryonic stem

Glucose-stimulated insulin secretion

Histone $\mathrm{H} 3$ at lysine 9

High-fat diet

Insulin tolerance test

$\mathrm{KCl} \quad$ Potassium chloride

KIC $\quad \alpha$-Ketoisocaproate

NIDDK National Institute of Diabetes and Digestive and Kidney Diseases

NIH National Institutes of Health

OCR Oxygen consumption rate 


$\begin{array}{ll}\text { ShRNA } & \text { Short hairpin RNA } \\ \text { SIRT6 } & \text { Sirtuin 6 } \\ \text { TEM } & \text { Transmission electron microscopy } \\ \text { TRPM2 } & \begin{array}{l}\text { Transient receptor potential cation channel } \\ \text { subfamily M, member } 2\end{array} \\ & \text { Wild-type }\end{array}$

\section{Introduction}

The pathogenesis of type 2 diabetes is multifactorial, but impaired insulin secretion from pancreatic beta cells is one of the critical factors [1]. Glucose-stimulated insulin secretion (GSIS) is a complex process that involves glucose sensing, transport and metabolism (glycolysis and mitochondrial oxidation), plasma membrane depolarisation, and calcium signalling and exocytosis, among other things [2].

Sirtuins belong to a conserved family of proteins, and mammals have seven members (SIRT1-7) [3]. SIRT6 is a chromatin-associated enzyme that deacetylates histone $\mathrm{H} 3$ at lysine 9 (H3K9) and lysine 56 residues [4-6]. Some nonhistone substrates, such as forkhead box O1 (FoxO1), general control of amino acid synthesis protein 5 (GCN5), and CTBPinteracting protein (CtIP), have also been reported [7-9]. SIRT6 can also remove long-chain fatty acyl groups from lysine residues of its substrates such as TNF- $\alpha[10,11]$. SIRT6-deficient mice exhibit accelerated ageing and die of hypoglycaemia by 4 weeks of age $[12,13]$. SIRT6 has been implicated in a variety of metabolic processes, including glycolysis, gluconeogenesis, hepatic lipid and cholesterol metabolism, neuroendocrine regulation and circadian regulation of metabolism [7, 14-21]. Interestingly, high-fat diet (HFD)treated Sirt6 transgenic mice secrete more insulin in response to a bolus of glucose than their wild-type (WT) counterparts [22]. These data suggest that SIRT6 is probably required for insulin secretion and beta cell function. In this work, we generated both pancreas- and beta cell-specific Sirt6 knockout mice to illustrate the role of SIRT6 in the pancreatic beta cells.

\section{Methods}

Animals Pancreas-specific deletion of Sirt6 was generated by crossing Sirt6 floxed mice Sirt $^{\mathrm{Tm} 1.1 \mathrm{Cxd}}$, provided by C. Deng, Mammalian Genetics Section, National Institute of Diabetes and Digestive and Kidney Disease [NIDDK], Bethesda, MD, USA) with $\operatorname{Tg}(\mathrm{Pdx} 1-\mathrm{Cre}) 6 \mathrm{Tuv}$ mice from the Jackson Laboratory (Bar Harbor, ME, USA) [16, 23]. Beta cell-specific Sirt6 deletion was generated by crossing Sirt6 floxed mice with MIP-Cre/ERT mice (Tg(Ins1-Cre/ ERT)1Lphi, provided by L. Philipson, Department of Medicine, University of Chicago, Chicago, IL, USA) and tamoxifen administration (oral gavage at a dose of $4 \mathrm{mg} /$ mouse in corn oil for four consecutive days) [24]. These mice were on the mixed background (FVB/NJ:129S6/Sv:C57BL/6J). Mice were fed either regular chow (18\% energy from fat) or a HFD ( $42 \%$ energy from fat, Harlan Teklad, Indianapolis, IN, USA). Blood glucose levels were measured under ad libitum or overnight $16 \mathrm{~h}$ fasting conditions. GTT and insulin tolerance test (ITT) were performed in mice fasted for 6 or $4 \mathrm{~h}$ before injection of glucose $(2 \mathrm{~g} / \mathrm{kg}$, i.p. or oral gavage $)$ or insulin $(0.5 \mathrm{U} / \mathrm{kg}$ for chow-fed mice and $0.75 \mathrm{U} / \mathrm{kg}$ for HFD-fed mice, i.p.), respectively. GSIS and L-arginine-stimulated insulin secretion were performed in mice fasted for $16 \mathrm{~h}$ before injection of glucose $(2 \mathrm{~g} / \mathrm{kg}$, i.p.) or L-arginine $(1 \mathrm{~g} / \mathrm{kg}$, i.p.), respectively. Tail-vein blood samples were collected for insulin measurements using an ELISA kit (ALPCO, Salem, $\mathrm{NH}$, USA). All animal procedures were performed in accordance with the National Institutes of Health (NIH) Guide for the Care and Use of Laboratory Animals and were approved by the Indiana University School of Medicine Institutional Animal Care and Use Committee. Samples were not randomised and the experimenters were not blind to group assignment and outcome assessment. No data were excluded for the report.

Cell culture MIN6 cells (provided by D. Thurmond, Department of Pediatrics, Indiana University, Indianapolis, IN, USA) were cultured and transduced with adenoviruses as previously described $[25,26]$. The cell line was verified in our laboratory and it did not have mycoplasma contamination.

Pancreatic islets Islets were isolated from mouse pancreases at the Islet Core of the Indiana Diabetes Research Center as previously described [27].

Insulin secretion analysis Insulin secretion analysis in MIN6 cells or mouse islets was performed as previously described [28]. Glucose, potassium chloride $(\mathrm{KCl}), \alpha$-ketoisocaproate (KIC, Sigma-Aldrich, St Louis, MO, USA) and ionomycin (IM, Cayman, Ann Arbor, MI, USA) were used for the experiments. Insulin was analysed using an ELISA kit (ALPCO).

RNA analysis Total RNA samples were prepared and analysed as previously described [25].

Protein analysis Protein extracts from mouse islets and other tissues or MIN6 cells were prepared and analysed as previously described [25]. The following antibodies were used: SIRT6 (Abcam, Cambridge, MA, USA, and Sigma-Aldrich, 1:1,000 dilution), actinin (Santa Cruz Biotechnology, Dallas, TX, USA, 1:1,000 dilution), Ac-H3K9 and cleaved caspase 3 (Cell Signaling Technology, Beverly, MA, USA, 1:1,000 dilution) and total OXPHOS antibody cocktail (Abcam, 1:250 dilution). Antibodies were validated through confirmation of 
protein molecular weight and their known characteristics according to existing knowledge.

Histological analysis Pancreases were fixed and processed as previously described [29]. The following antibodies were used for immunostaining: glucagon (Sigma-Aldrich, 1:5,000 dilution), insulin and Ki67 (Cell Signaling Technology, 1:500 dilution). Beta cell areas were determined by analysing 10-15 pancreatic sections stained for insulin per genotype using NIH ImageJ software (http://rsb.info.nih.gov/ij/download.html). To examine proliferating beta cells, 15-20 islets were analysed for each genotype. The number of Ki67-positive beta cells per islet was normalised to the total beta cell number in each islet.

Pancreatic insulin content analysis To measure total pancreatic insulin content, whole pancreas was dissected and processed as previously described [30]. Insulin was measured using an ELISA kit (ALPCO).

ATP analysis ATP was extracted from mouse islets using $2.5 \%$ trichloroacetic acid (wt/vol.) and measured using a bioluminescent assay kit (Sigma-Aldrich).

Cellular bioenergetics analysis The extracellular acidification rate (ECAR) and oxygen consumption rate (OCR) were measured in MIN6 cells using an XF24 Analyzer (Seahorse Bioscience, North Billerica, MA, USA).

Cytoplasmic $\mathrm{Ca}^{2+}$ analysis Cytosolic $\mathrm{Ca}^{2+}$ dynamics in mouse islets was measured using the Fura-2-AM (Invitrogen) calcium probe as previously described [31].

Electron microscopy Isolated mouse islets were fixed with $2 \%$ glutaraldehyde (wt/vol.) and processed as previously described [32]. Numbers of total mitochondria and damaged mitochondria from each cell were counted from a randomly selected group of 30 cells. The cytosolic area of each cell was determined using SPOT software (Sterling Heights, MI, USA), which was used to normalise the numbers of total and damaged mitochondria.

Statistical analysis All data are presented as means \pm SEM. Two-group comparisons were performed using two-tailed unpaired Student's $t$ test, and multiple-group comparisons were performed using ANOVA and Turkey's post hoc test. A $p$ value of $<0.05$ was considered as significant.

\section{Results}

SIRT6 regulates insulin secretion from pancreatic beta cells To examine Sirt6 gene expression in pancreatic islets, we performed real-time PCR and western blot analysis. The mRNA levels of Sirt6 in mouse islets were higher than that in brain, white adipose tissue, heart and liver (Fig. 1a). The western blot data also confirmed that SIRT6 protein was more readily detectable in mouse islets than in white adipose tissue, heart and liver (Fig. 1b). It is worth noting that the SIRT6 antibodies used also detected several other bands on the blot although their identities are unknown at this time. To assess the function of SIRT6 in pancreatic beta cells, we performed knockdown of the Sirt6 gene in a mouse insulinoma cell line, MIN6. Sirt6 knockdown was confirmed by western blot (Fig. 1c). As expected, acetylation of the H3K9 residue, a known deacetylation substrate of SIRT6, was elevated (Fig. 1c). SIRT6-deficient MIN6 cells secreted $30 \%$ less insulin compared with WT control cells treated with short hairpin (sh)RNAs against the green fluorescent protein gene (shGfp) when they were stimulated with $16.7 \mathrm{mmol} / 1$ glucose (Fig. 1d). These data suggest that SIRT6 regulates GSIS.

Pancreatic SIRT6 deficiency leads to insulin secretory impairment and glucose intolerance To further investigate the physiological role of SIRT6 in pancreatic beta cell development and function, we generated Sirt6 pancreas-specific knockout mice (bPko) by crossing mice bearing floxed Sirt6 alleles with $P d x 1$-Cre mice. The gene knockout was very efficient as indicated by western blot and quantitative PCR analysis (Fig. 2a, b). To assess whether glucose homeostasis was perturbed in the bPko mice, we first measured blood glucose in fasting and ad libitum-fed mice. No significant difference in blood glucose levels was noticed between the control and knockout mice (Fig. 2c). However, when the bPko mice were challenged with an i.p. or oral glucose load, they showed remarkable glucose intolerance (Fig. 2d, e). The impairment in glucose tolerance can be caused by a number of factors, including insulin resistance and insulin secretory defects. ITTs did not reveal any difference between the control and bPko mice (Fig. 2f). However, plasma insulin levels (balance of secretion and clearance) were reduced under basal and glucose-stimulated states in the bPko mice (Fig. 2g). The reduction of insulin secretion can be caused by a decrease in beta cell mass, insulin content or insulin secretion. Histological analysis by haematoxylin-eosin staining and immunostaining with antibodies against insulin and glucagon did not reveal any significant difference in islet shape or size, or distribution of alpha and beta cells between the bPko and control mice (Fig. 2h, i). In addition, beta cell area and pancreatic insulin content were not significantly different (Fig. 2j, k). These data suggest that insulin secretion defects might underlie the glucose intolerance in the bPko mice.

Deletion of Sirt6 in pancreatic beta cells causes insulin secretory defects Although $P d x 1$-Cre has been widely used to generate pancreas-specific knockout mice, the Cre 
a

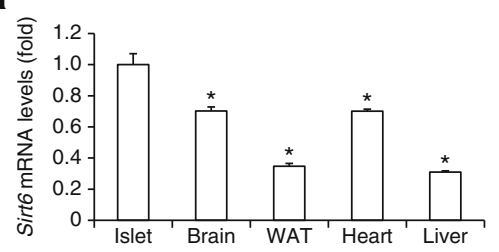

b

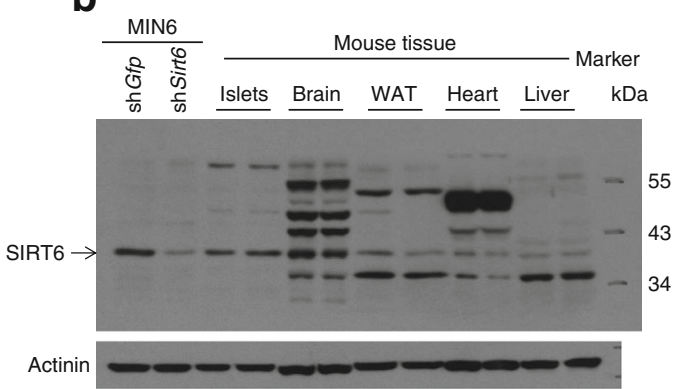

C

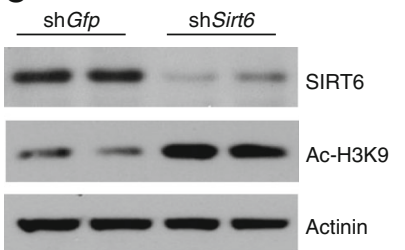

d

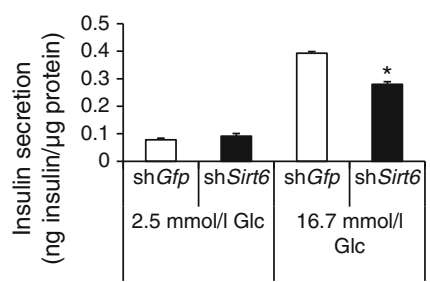

Fig. 1 Sirt6 knockdown impairs GSIS. (a) Sirt6 mRNA and (b) SIRT6 protein analysis in WT mouse islets and other tissues. (c) Immunoblot analysis and (d) GSIS in MIN6 cells transduced with shGfp and shSirt6 shRNA-expressing adenoviruses. Sirt6 mRNA levels in other tissues are presented as fold change to that in the islets. WAT, white adipose tissue; Glc, glucose. Data are presented as mean \pm SEM. ${ }^{*} p<0.05$ vs (a) islets; vs (d) $\operatorname{sh} G f p$

transgene also causes recombination in hypothalamus [24]. To investigate the role of SIRT6 in adult beta cells, we took advantage of a tamoxifen-inducible $M I P$-Cre/ERT mouse strain. The MIP-Cre/ERT mouse exhibits no leakage of Cre expression in the brain and is considered to be pancreatic beta cellspecific [24]. We first tested the knockout efficiency in the islets from Sirt6 ${ }^{\text {lox/lox}}:$ MIP-Cre/ERT knockout (bMko) mice. Sirt6 was efficiently deleted 2 weeks after tamoxifen administration as shown by western blot analysis and quantitative PCR of mouse islets (Fig. 3a, b). Fasting and ad libitum-fed blood glucose levels were similar in bMko, floxed (loxp) and Cre mice (Fig. 3c). However, the bMko mice showed significant glucose intolerance compared with control mice (Fig. 3d). Like the bPko mice, the bMko mice also did not exhibit any alteration in insulin tolerance (Fig. 3e). Remarkably, the bMko mice displayed significantly lower plasma insulin levels than the control mice after a glucose load (Fig. 3f).

SIRT6 deficiency impairs mitochondrial glucose oxidation To examine whether or not the decrease in GSIS in SIRT6-
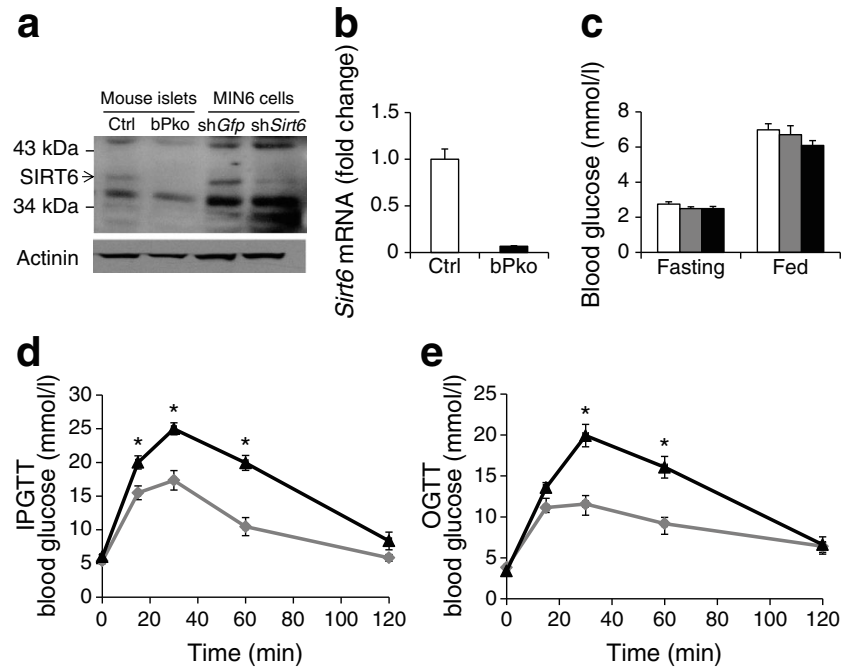

f

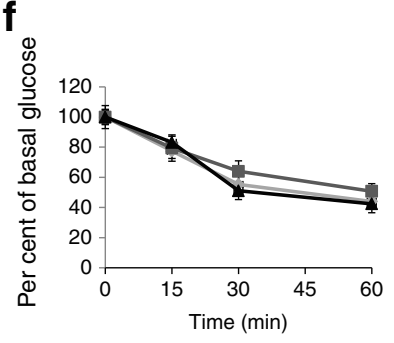

g

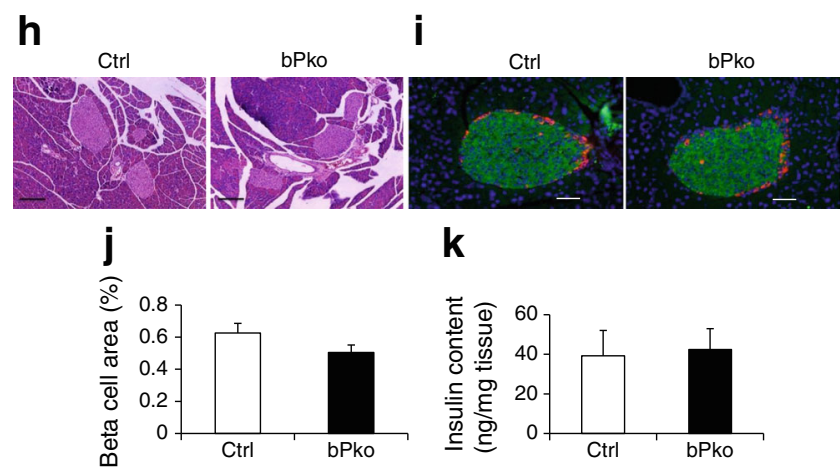

Fig. 2 Impaired glucose tolerance and GSIS in bPko mice. (a) SIRT6 protein and (b) Sirt6 mRNA analysis in control (Ctrl) and bPko islets (MIN6 cells as controls). (c) Blood glucose, (d) i.p. GTT (IPGTT), (e) OGTT, (f) ITT and (g) GSIS in 2-3-month-old male mice ( $n=6-7$ each genotype; loxp mice, white bar or light grey diamond; Cre mice, dark grey bar or square; bPko mice, black bar or triangle). Pancreatic sections stained with (h) haematoxylin-eosin and (i) insulin (green) and glucagon antibodies (red); (j) beta cell area and (k) pancreatic insulin content of control and bPko mice $(n=3)$. Data are presented as mean \pm SEM. ${ }^{*} p<0.05$ vs Ctrl. Scale bars, (h) $100 \mu \mathrm{m}$, (i) $50 \mu \mathrm{m}$

deficient islets is intrinsic to pancreatic beta cells, we performed ex vivo GSIS analysis using isolated mouse islets. Although there was no difference in insulin secretion at a low concentration of glucose $(2.5 \mathrm{mmol} / \mathrm{l})$ between bMko and control islets (Fig. 4a), the amount of insulin secreted in response to a high concentration of glucose $(16.7 \mathrm{mmol} / \mathrm{l})$ was reduced by $46 \%$ in the bMko islets compared with controls (Fig. 4a). 
a
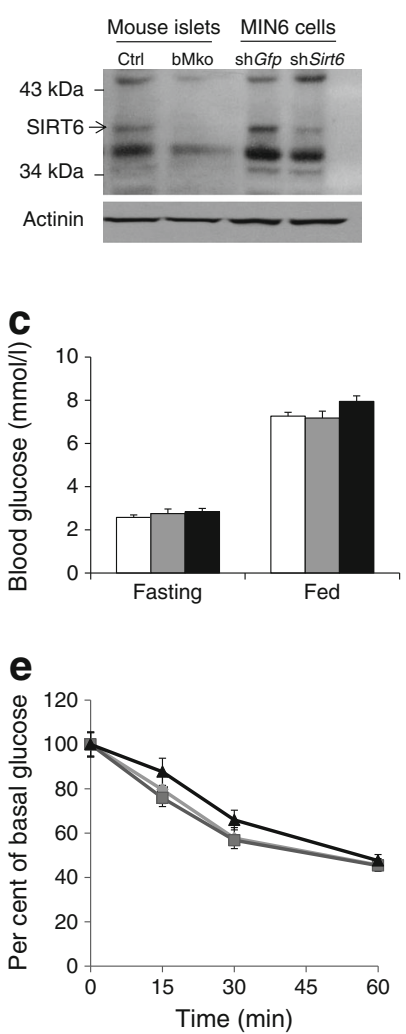
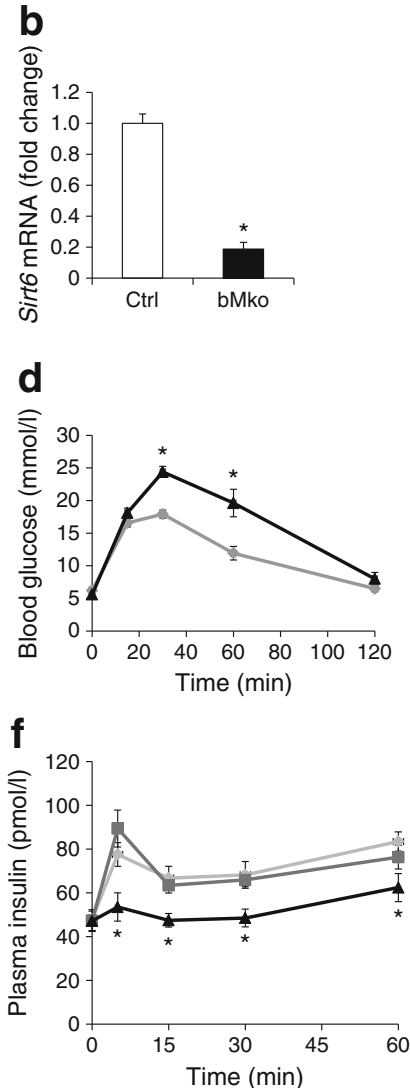

Fig. 3 Sirt6 beta cell-specific deletion causes impaired GSIS. (a) SIRT6 protein and (b) Sirt6 mRNA analysis in control (Ctrl) and bMko islets (MIN6 cells used as controls). (c) Blood glucose, (d) i.p. GTT, (e) ITT and (f) GSIS in 3-month-old male mice $(n=6-7$ each genotype; loxp mice, white bar or light grey diamond; Cre mice, dark grey bar or square; bMko mice, black bar or triangle). Data are presented as mean \pm SEM. $* p<0.05$ vs Ctrl

To identify the potential causes for the GSIS defects in SIRT6-deficient beta cells, we first examined glucose metabolism in MIN6 cells using an extracellular flux analyser (Seahorse; mouse islets could not be examined because of technical issues). Glycolysis and mitochondrial respiration can be monitored by measuring ECAR and OCR, respectively. Under high glucose conditions, Sirt6 knockout significantly reduced the levels of ECAR and OCR in MIN6 cells, indicating a decrease of glucose metabolism (Fig. 4b, c). Next, we measured ATP production, which is the final product of mitochondrial glucose metabolism and controls insulin secretion via closure of $\mathrm{K}_{\mathrm{ATP}}$ channels. At low glucose levels ( $2.5 \mathrm{mmol} / \mathrm{l}), \mathrm{bMko}$ and control islets had comparable levels of ATP; however, the ATP production in response to $16.7 \mathrm{mmol} / \mathrm{l}$ glucose was decreased by $\sim 20 \%$ in the bMko islets relative to controls (Fig. 4d). To further confirm the defect in mitochondrial energy metabolism, we used KIC, a mitochondrial substrate that can be directly metabolised by the tricarboxylic acid cycle and bypass glycolysis to perform insulin secretion assays in isolated bMko and control islets. As predicted, bMko islets secreted 58\% less insulin after

a

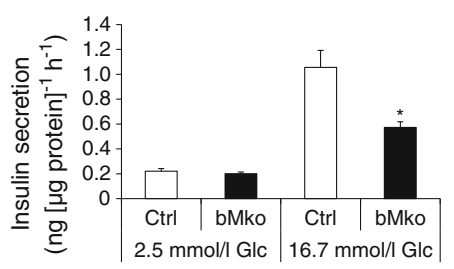

b
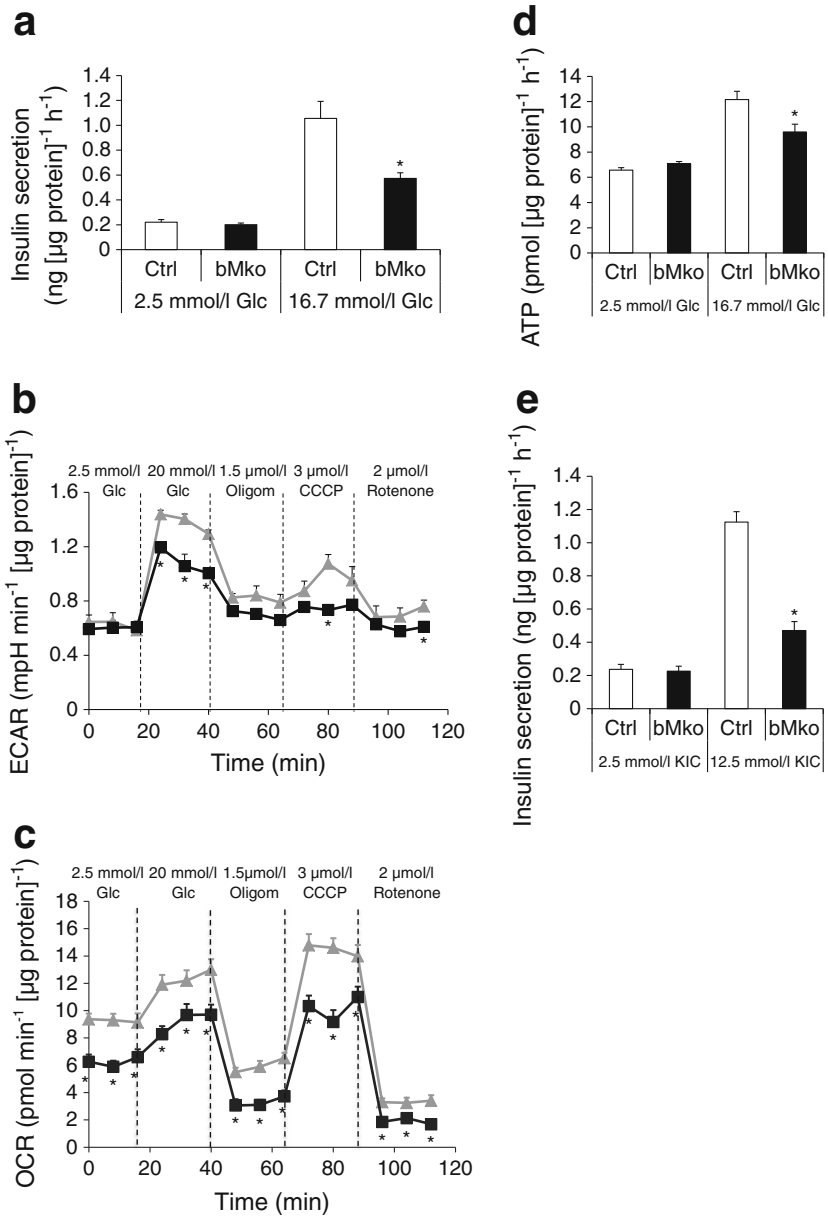

Fig. 4 SIRT6 deficiency in beta cells leads to impaired glucose oxidation. (a) GSIS analysis in control (Ctrl) and bMko islets. (b) ECAR and (c) OCR analysis in MIN6 cells transduced with shGfp (triangle) and shSirt6 (square) adenoviruses and treated with glucose (Glc), oligomycin (Oligom), carbonyl cyanide 3-chlorophenylhydrazone (CCCP) and rotenone. (d) Glucose-stimulated ATP production in control and bMko islets. (e) KIC-stimulated insulin secretion in Ctrl and bMko islets. Each experiment was performed with pooled islets from three mice per genotype. Data are presented as mean \pm SEM, $n=3 .{ }^{*} p<0.05$ vs (a, d, e) Ctrl, vs $(\mathbf{b}, \mathbf{c}) \operatorname{sh} G f p$

incubation with $12.5 \mathrm{mmol} / \mathrm{l} \mathrm{KIC} \mathrm{compared} \mathrm{with} \mathrm{controls}$ (Fig. 4e), suggesting a defect in mitochondrial oxidation.

Ablation of SIRT6 in pancreatic beta cells leads to mitochondrial defects To examine what caused the impairment of glucose oxidation in mitochondria, we surveyed five mitochondrial complexes that are involved in the electron transport chain using a cocktail of antibodies against representative proteins from Complexes I to V. Western blots and densitometry analysis revealed that levels of Complexes III and IV were significantly decreased in the bMko mouse islets compared with controls (Fig. 5a, b). This suggests that Sirt6 gene deletion causes mitochondrial deficiency. In order to visualise mitochondria in structural detail, we also preformed transmission electron microscopy (TEM). By reviewing the 
a

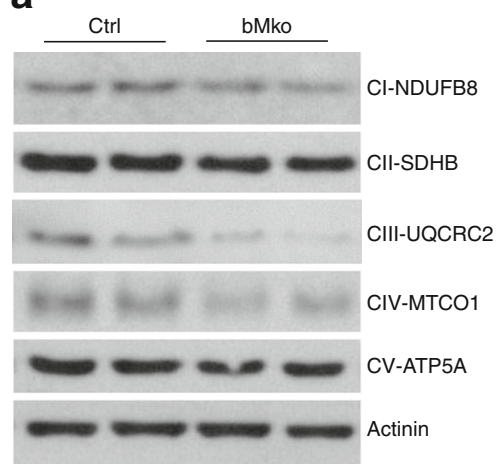

b

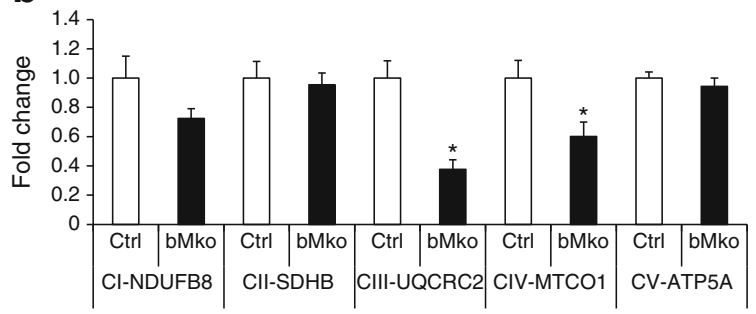

Fig. 5 Pancreatic beta cell Sirt6 knockout leads to deficiency in the mitochondrial electron transport chain. (a) Immunoblot and (b) quantitative analysis of representative proteins in mitochondrial Complexes I-V in primary islets isolated from 4-month-old control (Ctrl) and bMko mice $(n=6)$. Protein signal intensity was normalised to loading control actinin. CI-NDUFB8, Complex I-NADH dehydrogenase 1 beta subcomplex subunit 8; CII-SDHB, Complex II-succinate dehydrogenase complex subunit B; CIII-UQCRC2, Complex III-ubiquinol-cytochrome c reductase core protein II; CIV-MTCO1, Complex IV-mitochondrially encoded cytochrome c oxidase I; CV-ATP5A, Complex V-ATP synthase mitochondrial F1 complex alpha subunit. Data are presented as mean \pm SEM, $n=6$. ${ }^{*} p<0.05$ vs Ctrl

TEM images, we observed noticeable mitochondrial damage in Sirt6-knockout beta cells (Fig. 6a-c). While total numbers of mitochondria were not different between control and SIRT6-deficient beta cells (Fig. 6d), numbers of damaged mitochondria increased in the Sirt6-knockout beta cells compared with the control counterparts (Fig. 6e).

\section{SIRT6 deficiency causes aberrant calcium flux in beta cells} To further examine whether or not SIRT6 deficiency might alter membrane depolarisation and calcium flux in pancreatic beta cells, we performed insulin release analysis after L-arginine injections in mice. L-Arginine is known to depolarise the plasma membrane by its cationic charges. Plasma insulin levels were $\sim 50 \%$ lower in the bMko mice compared with the control group 2 min after L-arginine injections (Fig. 7a). Moreover, we performed ex vivo $\mathrm{KCl}$-stimulated insulin secretion in isolated mouse islets, as $\mathrm{KCl}$ also has a strong membrane depolarisation effect. Insulin secretion in response to $30 \mathrm{mmol} / \mathrm{l} \mathrm{KCl}$ was decreased $50 \%$ in the bMko islets compared with the WT controls (Fig. 7b). These data suggest that the defective insulin secretion might also result from defects in either plasma membrane depolarisation and/or post-depolarisation. a

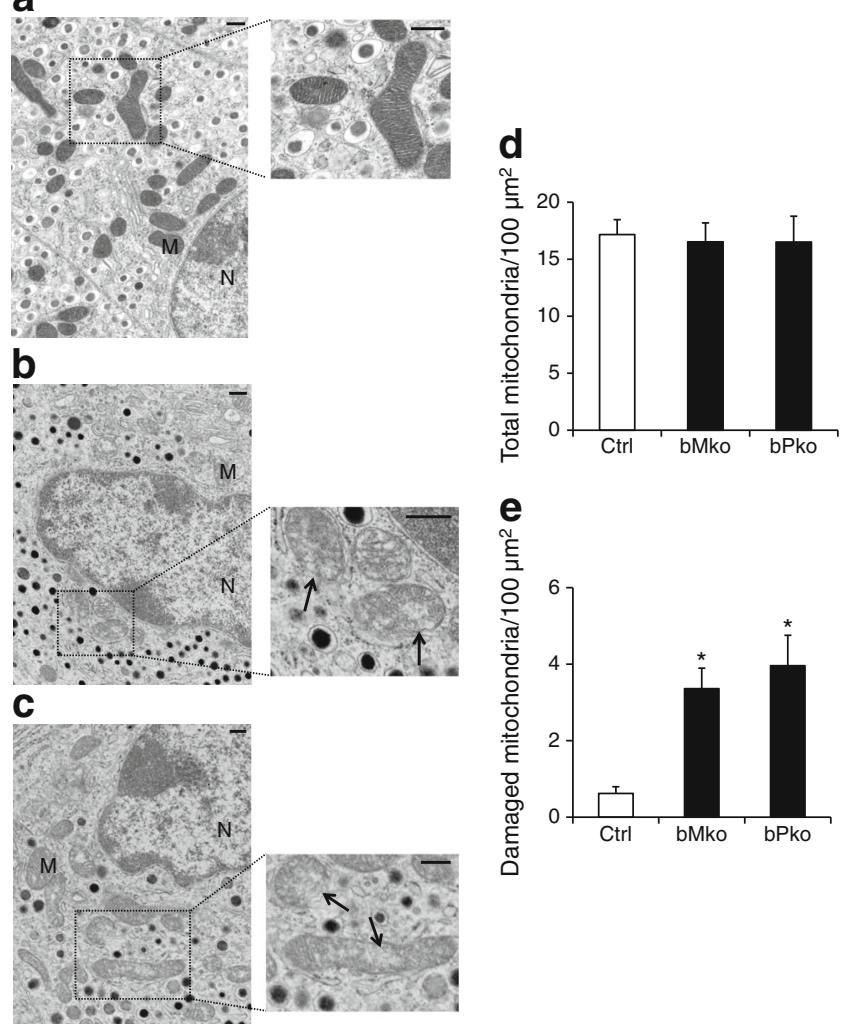

Fig. 6 Beta cell SIRT6 deficiency increases the number of damaged mitochondria. Representative electron microscopy images $(\times 3,000$ magnification) of isolated islets from (a) 3-month control (Ctrl), (b) bMko and (c) bPko mice. Enlargements of areas are shown to the right. Arrows denote damaged mitochondria. Numbers of (d) total and (e) damaged mitochondria were quantified in beta cells $(n=20-30)$. M, mitochondria; $\mathrm{N}$, nuclei. Data are presented as mean $\pm \mathrm{SEM}$. ${ }^{*} p<0.05$ vs Ctrl. Scale bars, $500 \mathrm{~nm}$

In pancreatic beta cells, an increase in intracellular calcium $\left(\left[\mathrm{Ca}^{2+}\right]_{\mathrm{i}}\right)$ is critical for secretagogue-induced insulin release $[33,34]$. To examine whether the reduction in the insulin secretory response to glucose or $\mathrm{KCl}$ may be associated with reduced calcium influx, we measured $\left[\mathrm{Ca}^{2+}\right]_{\mathrm{i}}$ (indicated by the fluorescence ratio of $340 \mathrm{~nm} / 380 \mathrm{~nm}$ ) of isolated islets loaded with Fura-2-AM fluorescent probes. Consistent with the observation that bMko mice had normal basal insulin secretion, the islets from bMko and control mice showed similar resting $\left[\mathrm{Ca}^{2+}\right]_{\mathrm{i}}$ levels (Fig. $7 \mathrm{c}, \mathrm{d}$ ). However, in response to $16.7 \mathrm{mmol} /$ 1 glucose or $30 \mathrm{mmol} / 1 \mathrm{KCl},\left[\mathrm{Ca}^{2+}\right]_{\mathrm{i}}$ was significantly lower in the bMko islets compared with the control group (Fig. 7c, d), suggesting the presence of a defect in calcium flux regulation. To further verify that the reduction of $\left[\mathrm{Ca}^{2+}\right]_{\mathrm{i}}$ is critical for the impaired insulin secretion in SIRT6-deficient beta cells, we performed insulin secretion assays in bMko and control islets in the presence of a $\mathrm{Ca}^{2+}$ ionophore, ionomycin. Remarkably, $30 \mu \mathrm{mol} / 1$ ionomycin rescued the insulin secretion deficiency in the bMko islets when stimulated with $16.7 \mathrm{mmol} / \mathrm{l}$ glucose (Fig. 7e). These data suggest that SIRT6 regulates calcium flux in the pancreatic beta cells. 

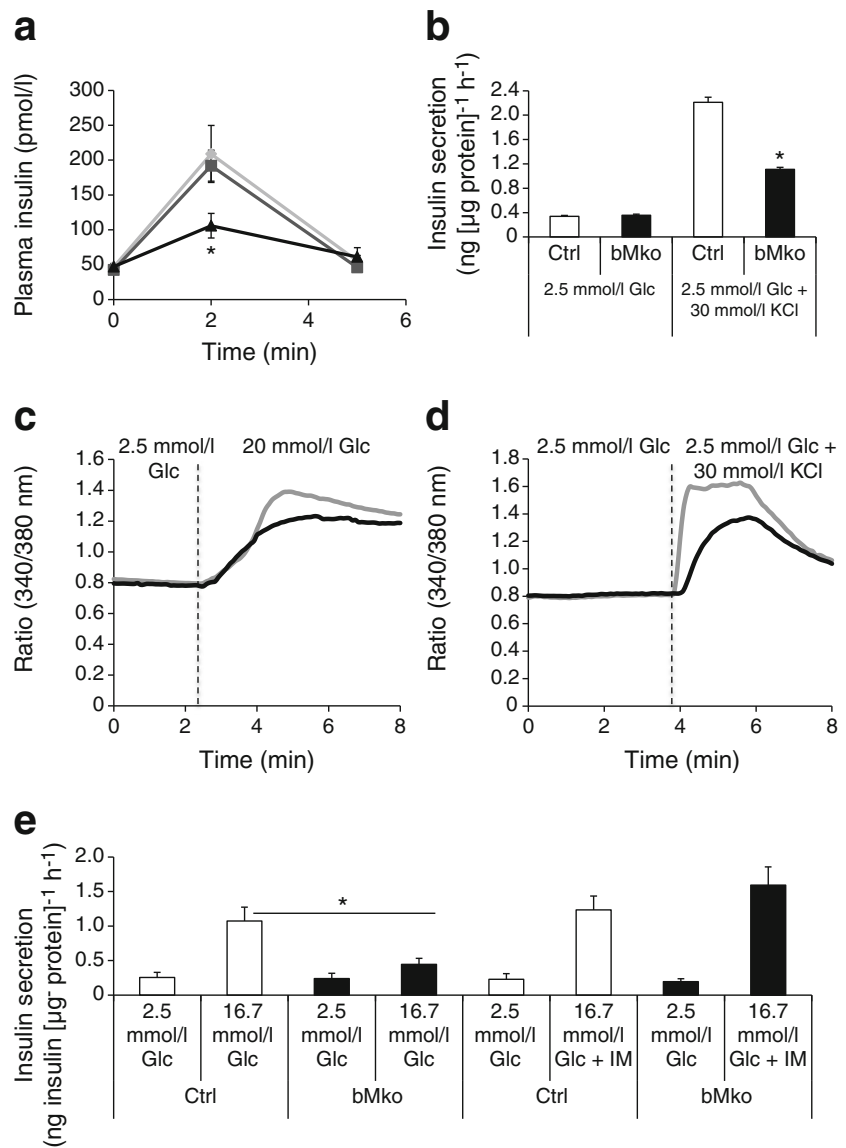

Fig. 7 Inactivation of the Sirt6 gene in beta cells causes impaired calcium flux. L-Arginine-stimulated insulin release (a) in 3-month-old male loxp (diamond), MIP-Cre (square) and bMko (triangle) mice ( $n=6$ per group). $\mathrm{KCl}$-stimulated insulin secretion (b) in control (Ctrl) and bMko islets. Representative traces of cytosolic $\mathrm{Ca}^{2+}$ flux measured by Fura2-AM loading in control (grey lines) and bMko (black lines) islets incubated with (c) glucose (Glc) and (d) KCl, respectively. (e) Insulin secretion from control and bMko islets treated with 2.5 or $16.7 \mathrm{mmol} / \mathrm{l} \mathrm{Glc}$ alone or in combination with $30 \mu \mathrm{mol} / \mathrm{l}$ ionomycin (IM). Data are presented as mean \pm SEM, $n=3 .{ }^{*} p<0.05$ vs Ctrl

Pancreatic beta cell SIRT6 is required to protect mice against obesity-induced glucose intolerance To examine the role of SIRT6 in pancreatic beta cells under obese conditions, we treated bMko and control mice with a 42\% HFD for 4 months. After HFD feeding, fasting blood glucose levels were comparable between the bMko and control mice (Fig. 8a), whereas fed blood glucose levels were increased in the bMko mice although they did not reach statistical significance (Fig. 8a). Glucose intolerance was worse in the bMko mice than that in the control group (Fig. 8b). Insulin tolerance was not significantly different between the bMko and control mice (Fig. 8c). Furthermore, bMko mice exhibited greater impairment in glucose-stimulated insulin release than control mice (Fig. 8d). To investigate whether or not SIRT6 plays a role in compensatory hyperplasia of beta cells induced by the HFD feeding, beta cell area and pancreatic insulin content were analysed. There were no significant differences in the
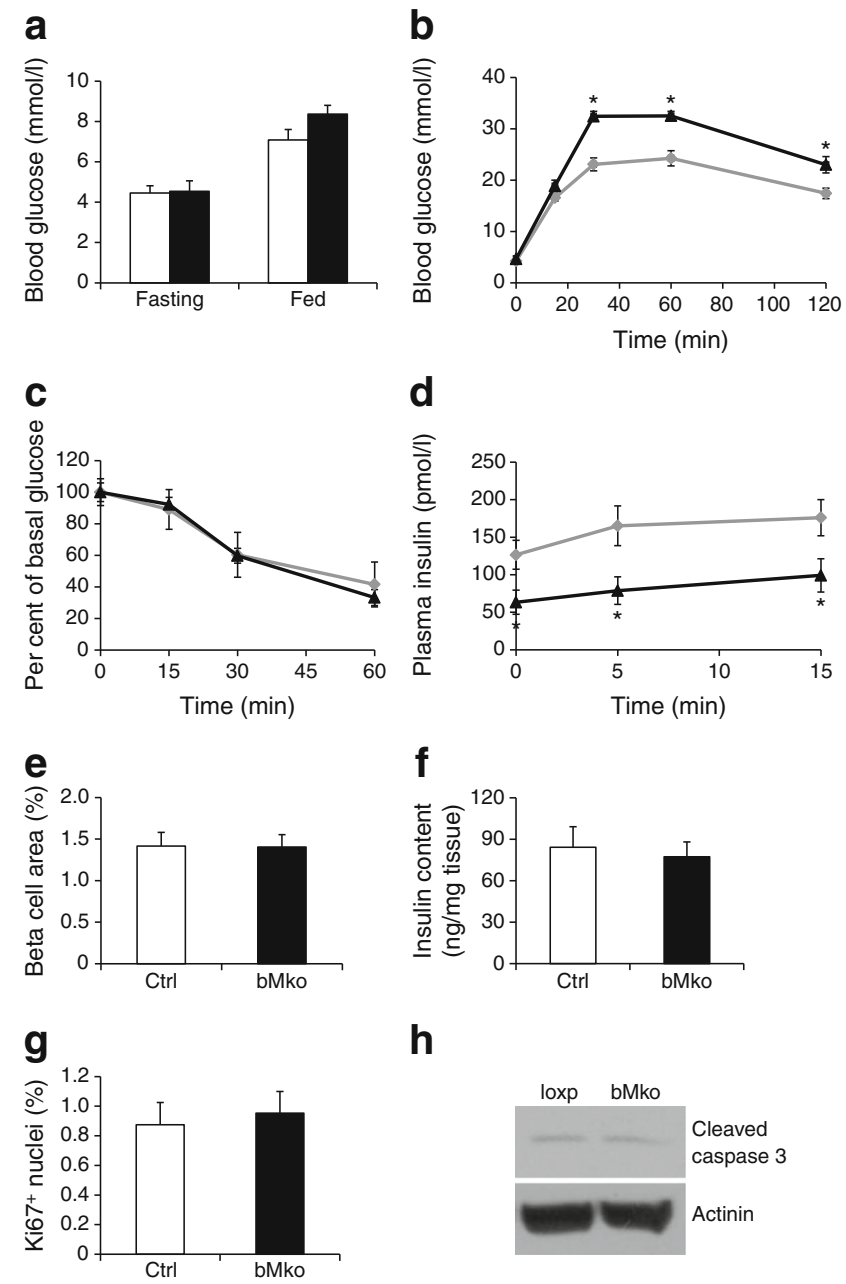

h

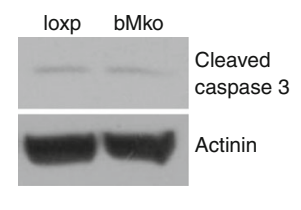

Fig. 8 Worsening of glucose intolerance and GSIS in the bMko mice on HFD. (a) Blood glucose, (b) i.p. GTT, (c) ITT and (d) GSIS in control (Ctrl, white bars/grey lines) and bMko (black bars/lines) male mice treated with 42\% HFD for 3 months $(n=7-8)$. (e) Beta cell area $(n=5)$, (f) pancreatic insulin content $(n=5),(\mathbf{g}) \mathrm{Ki} 67$-positive cells $(n=5)$ and (h) a representative immunoblot for cleaved caspase $3(n=4) \mathrm{Ctrl}$ and bMko mice after 4-month HFD feeding. Data are presented as mean \pm SEM. $* p<0.05$ vs Ctrl

beta cell area and pancreatic insulin content between the bMko and control mice (Fig. 8e, f). Moreover, beta cell proliferation, as measured by Ki67 staining of pancreatic sections, was comparable between the bMko and control mice (Fig. 8g). In addition, analysis of cleaved caspase 3 (a marker of apoptosis) in isolated islets showed that Sirt6 deletion did not increase caspase 3 activation even after HFD treatment (Fig. 8h).

\section{Discussion}

Impairment of GSIS is one of the early clinical manifestations in the development of type 2 diabetes [35]; however, the underlying mechanisms are not well understood. In this work, 
we have shown that SIRT6 is required for proper insulin secretion in response to glucose stimulation. Our data suggest that SIRT6 regulates GSIS through mitochondrial glucose oxidation, plasma membrane depolarisation and calcium dynamics (and possibly other mechanisms).

SIRT6 is expressed in multiple tissues, albeit at different levels with high expression in the skeletal muscle, thymus and brain in mice [12]. Our data show here that SIRT6 is readily detectable in mouse islets. Data from a previous transcriptomic analysis of purified mouse pancreatic alpha and beta cells also reveal that Sirt6 gene expression ranks in approximately the 75th and 80th percentiles among 23,406 genes in alpha and beta cells, respectively [36]. By contrast, another SIRT family member, SIRT1, which has been implicated in the regulation of insulin secretion from pancreatic beta cells [37-40], only ranks in the 68th and 64th percentiles in alpha and beta cells, respectively [36]. These data suggest that SIRT6 is likely to play a role in beta cell function.

SIRT6 has been shown to suppress glycolysis in mouse embryonic stem (ES) cells, fibroblasts and hepatocytes [16, 21]. However, this does not seem to be the case in pancreatic beta cells because glycolysis was decreased in SIRT6-deficient MIN6 cells in response to high glucose. Interestingly, the ECAR was decreased in both WT and SIRT6-deficient MIN6 cells in the presence of oligomycin, which usually inhibits mitochondrial OCR and promotes ECAR. This finding suggests that those beta cells might have low capacity to convert pyruvate to lactate compared with other cell types. Our data are consistent with a recent report in mouse primary islets [41]. It is well known that an increase in ATP levels or the ATP/ADP ratio from glucose metabolism is a critical trigger in GSIS [2]. Significantly, in our study, ATP production in the bMko islets upon glucose stimulation was lower than that in the WT islets. This result can be attributed to the compromised mitochondrial oxidation as indicated by reduced OCR in the SIRT6-deficient beta cells. Interestingly, levels of mitochondrial Complexes III and IV were decreased in the Sirt6 knockout beta cells. The electron microscopy analysis also reveals an increase in mitochondrial damage in the SIRT6-deficient beta cells. However, the cause of the mitochondrial defects is unclear. Consistent with these findings, mitochondrial defects have been also observed in Sirt6-knockout mouse ES cells and Sirt6-knockout breast cancer cells [21, 42]. In SIRT6-deficient mouse ES cells, mitochondrial respiration and a number of intermediate metabolites in the tricarboxylic acid cycle, including citrate, isocitrate, succinate, fumarate and malate, are decreased [21]. In Hs578t breast cancer cells, overexpression of SIRT6 increases OCR and knockdown of SIRT6 decreases it [42]. Together, these data suggest that SIRT6 promotes mitochondrial respiration. However, further study is required to elucidate how SIRT6 regulates mitochondrial function.

A high concentration of $\mathrm{KCl}$ can cause depolarisation of the beta cell plasma membrane, which subsequently triggers $\mathrm{Ca}^{2+}$ influx and insulin granule exocytosis [33]. The reduction in $\mathrm{Ca}^{2+}$ influx and insulin secretion from the bMko mouse islets in response to $30 \mathrm{mmol} / 1 \mathrm{KCl}$ seen in the current study suggests that SIRT6 may regulate insulin secretion at membrane depolarisation and/or downstream of the depolarisation event. According to our data, aberrant $\mathrm{Ca}^{2+}$ flux is one of the potential downstream defects in SIRT6-deficient beta cells. The first evidence is that cytosolic $\left[\mathrm{Ca}^{2+}\right]$ was lower in bMko islets in response to high glucose or $\mathrm{KCl}$ compared with the $\mathrm{WT}$ islets. Second, the increase of cytosolic $\left[\mathrm{Ca}^{2+}\right]$ caused by the $\mathrm{Ca}^{2+}$ ionophore ionomycin normalised GSIS in the bMko islets. Since ionomycin has multiple actions that increase cytosolic $\left[\mathrm{Ca}^{2+}\right]$, including store-operated $\mathrm{Ca}^{2+}$ entry and calciuminduced $\mathrm{Ca}^{2+}$ release [43], the precise mechanism of the regulation by SIRT6 is not clear yet. In addition, transient receptor potential cation channel, subfamily M, member 2 (TRPM2) has been suggested to play a role in insulin secretion $[44,45]$, and SIRT6 can modulate TRPM2 activity through its byproduct $O$ acetyl-ADP ribose (OAADPR) and its derivative ADP ribose (ADPR) [46], which can activate TRPM2 [44]. It would be interesting to see that to what extent the OAADPR/ADPRTRPM2 pathway contributes to the SIRT6 effect on GSIS.

In summary, this work characterises the role of SIRT6 in pancreatic beta cells and reveals its importance in insulin secretion and glucose homeostasis. Specifically, our data demonstrate that SIRT6 activity is necessary to regulate insulin secretion by maintaining mitochondrial function and modulating $\mathrm{Ca}^{2+}$ dynamics. Therefore, it is important to further investigate the mechanisms by which SIRT6 regulates beta cell function. Pharmacological activation of SIRT6 may be useful to enhance insulin secretion and it has potential for the development of effective drugs to treat type 2 diabetes.

Acknowledgements We thank C. Deng (NIDDK, Bethesda, MD, USA) for providing the Sirt6 floxed mice, L. Philipson (University of Chicago, Chicago, IL, USA) for providing the MIP-Cre mice, D. Thurmond (Indiana University School of Medicine, Indianapolis, IN, USA) for providing the MIN6 cells, and P. Fueger, B. Maier and E. Oh (Indiana University School of Medicine, Indianapolis, IN, USA) for technical discussions.

Funding This work was supported by grant no. R01DK091592 (XCD) from the NIDDK.

Duality of interest The authors declare that there is no duality of interest associated with this manuscript.

Contribution statement XX carried out the study, interpreted and analysed the data, and wrote the manuscript. GW, RT, PW, KL, TK, XT and SAT contributed to data collection and manuscript preparation and revision. RAH, CE-M and RGM participated in the experimental design, data interpretation, and manuscript preparation and revision. W-XD contributed to data collection and interpretation and manuscript writing. $\mathrm{XCD}$ conceived the hypothesis, designed the experiments, analysed and interpreted the data and wrote the manuscript. XCD is the guarantor of this work. All authors approved the manuscript. 


\section{References}

1. Prentki M, Nolan CJ (2006) Islet beta cell failure in type 2 diabetes. J Clin Invest 116:1802-1812

2. Prentki M, Matschinsky FM, Madiraju SR (2013) Metabolic signaling in fuel-induced insulin secretion. Cell Metab 18:162-185

3. Dong XC (2012) Sirtuin biology and relevance to diabetes treatment. Diabetes Manag (Lond) 2:243-257

4. Michishita E, McCord RA, Berber E et al (2008) SIRT6 is a histone H3 lysine 9 deacetylase that modulates telomeric chromatin. Nature 452:492-496

5. Michishita E, McCord RA, Boxer LD et al (2009) Cell cycledependent deacetylation of telomeric histone $\mathrm{H} 3$ lysine $\mathrm{K} 56$ by human SIRT6. Cell Cycle 8:2664-2666

6. Yang B, Zwaans BM, Eckersdorff M, Lombard DB (2009) The sirtuin SIRT6 deacetylates H3 K56Ac in vivo to promote genomic stability. Cell Cycle 8:2662-2663

7. Dominy JE Jr, Lee Y, Jedrychowski MP et al (2012) The deacetylase Sirt6 activates the acetyltransferase GCN5 and suppresses hepatic gluconeogenesis. Mol Cell 48:900-913

8. Kaidi A, Weinert BT, Choudhary C, Jackson SP (2010) Human SIRT6 promotes DNA end resection through CtIP deacetylation. Science 329:1348-1353

9. Zhang P, Tu B, Wang H et al (2014) Tumor suppressor p53 cooperates with SIRT6 to regulate gluconeogenesis by promoting FoxO1 nuclear exclusion. Proc Natl Acad Sci U S A 111:10684-10689

10. Jiang H, Khan S, Wang Y et al (2013) SIRT6 regulates TNF-alpha secretion through hydrolysis of long-chain fatty acyl lysine. Nature 496:110-113

11. Feldman JL, Baeza J, Denu JM (2013) Activation of the protein deacetylase SIRT6 by long-chain fatty acids and widespread deacylation by mammalian sirtuins. J Biol Chem 288:31350-31356

12. Mostoslavsky R, Chua KF, Lombard DB et al (2006) Genomic instability and aging-like phenotype in the absence of mammalian SIRT6. Cell 124:315-329

13. Xiao C, Kim HS, Lahusen Tet al (2010) SIRT6 deficiency results in severe hypoglycemia by enhancing both basal and insulinstimulated glucose uptake in mice. J Biol Chem 285:36776-36784

14. Masri S, Rigor P, Cervantes M et al (2014) Partitioning circadian transcription by SIRT6 leads to segregated control of cellular metabolism. Cell 158:659-672

15. Elhanati S, Kanfi Y, Varvak A et al (2013) Multiple regulatory layers of SREBP1/2 by SIRT6. Cell Rep 4:905-912

16. Kim HS, Xiao C, Wang RH et al (2010) Hepatic-specific disruption of SIRT6 in mice results in fatty liver formation due to enhanced glycolysis and triglyceride synthesis. Cell Metab 12:224-236

17. Schwer B, Schumacher B, Lombard DB et al (2010) Neural sirtuin 6 (Sirt6) ablation attenuates somatic growth and causes obesity. Proc Natl Acad Sci U S A 107:21790-21794

18. Tao R, Xiong X, DePinho RA, Deng CX, Dong XC (2013) FoxO3 transcription factor and Sirt6 deacetylase regulate low density lipoprotein (LDL)-cholesterol homeostasis via control of the proprotein convertase subtilisin/kexin type 9 (Pcsk9) gene expression. J Biol Chem 288:29252-29259

19. Tao R, Xiong X, DePinho RA, Deng CX, Dong XC (2013) Hepatic SREBP-2 and cholesterol biosynthesis are regulated by FoxO3 and Sirt6. J Lipid Res 54:2745-2753

20. Xiong X, Tao R, DePinho RA, Dong XC (2013) Deletion of hepatic FoxO1/3/4 genes in mice significantly impacts on glucose metabolism through downregulation of gluconeogenesis and upregulation of glycolysis. PLoS One 8:e74340

21. Zhong L, D'Urso A, Toiber D et al (2010) The histone deacetylase Sirt6 regulates glucose homeostasis via Hiflalpha. Cell 140:280-293
22. Kanfi Y, Peshti V, Gil R et al (2010) SIRT6 protects against pathological damage caused by diet-induced obesity. Aging Cell 9:162-173

23. Hingorani SR, Petricoin EF, Maitra A et al (2003) Preinvasive and invasive ductal pancreatic cancer and its early detection in the mouse. Cancer Cell 4:437-450

24. Wicksteed B, Brissova M, Yan W et al (2010) Conditional gene targeting in mouse pancreatic ss-cells: analysis of ectopic Cre transgene expression in the brain. Diabetes 59:3090-3098

25. Xiong X, Tao R, DePinho RA, Dong XC (2012) The autophagyrelated gene 14 (Atg14) is regulated by forkhead box O transcription factors and circadian rhythms and plays a critical role in hepatic autophagy and lipid metabolism. J Biol Chem 287:39107-39114

26. Kalwat MA, Wiseman DA, Luo W, Wang Z, Thurmond DC (2012) Gelsolin associates with the $\mathrm{N}$ terminus of syntaxin 4 to regulate insulin granule exocytosis. Mol Endocrinol 26:128-141

27. Stull ND, Breite A, McCarthy R, Tersey SA, Mirmira RG (2012) Mouse islet of Langerhans isolation using a combination of purified collagenase and neutral protease. J Vis Exp (67), e4137, doi:10. 3791/4137

28. Fueger PT, Hernandez AM, Chen YC, Colvin ES (2012) Assessing replication and beta cell function in adenovirally-transduced isolated rodent islets. J Vis Exp (64), e4080, doi:10.3791/4080

29. Kono T, Ahn G, Moss DR et al (2012) PPAR-gamma activation restores pancreatic islet SERCA2 levels and prevents beta-cell dysfunction under conditions of hyperglycemic and cytokine stress. Mol Endocrinol 26:257-271

30. Antkowiak PF, Tersey SA, Carter JD et al (2009) Noninvasive assessment of pancreatic beta-cell function in vivo with manganese-enhanced magnetic resonance imaging. Am J Physiol Endocrinol Metab 296:E573-E578

31. Johnson JS, Kono T, Tong X et al (2014) Pancreatic and duodenal homeobox protein $1(\mathrm{Pdx}-1)$ maintains endoplasmic reticulum calcium levels through transcriptional regulation of sarco-endoplasmic reticulum calcium ATPase 2b (SERCA2b) in the islet beta cell. J Biol Chem 289:32798-32810

32. Ding WX, Guo F, Ni HM et al (2012) Parkin and mitofusins reciprocally regulate mitophagy and mitochondrial spheroid formation. J Biol Chem 287:42379-42388

33. Gilon P, Chae HY, Rutter GA, Ravier MA (2014) Calcium signaling in pancreatic beta-cells in health and in type 2 diabetes. Cell Calcium 56:340-361

34. Yang SN, Berggren PO (2006) The role of voltage-gated calcium channels in pancreatic beta-cell physiology and pathophysiology. Endocr Rev 27:621-676

35. Weir GC, Bonner-Weir S (2004) Five stages of evolving beta-cell dysfunction during progression to diabetes. Diabetes 53(Suppl 3):S16-S21

36. Benner C, van der Meulen T, Caceres E, Tigyi K, Donaldson CJ, Huising MO (2014) The transcriptional landscape of mouse beta cells compared to human beta cells reveals notable species differences in long non-coding RNA and protein-coding gene expression. BMC Genomics 15:620

37. Bordone L, Motta MC, Picard F et al (2006) Sirt1 regulates insulin secretion by repressing UCP2 in pancreatic beta cells. PLoS Biol 4:e31

38. Luu L, Dai FF, Prentice KJ et al (2013) The loss of Sirt1 in mouse pancreatic beta cells impairs insulin secretion by disrupting glucose sensing. Diabetologia 56:2010-2020

39. Moynihan KA, Grimm AA, Plueger MM et al (2005) Increased dosage of mammalian Sir2 in pancreatic beta cells enhances glucose-stimulated insulin secretion in mice. Cell Metab 2:105-117

40. Pinho AV, Bensellam M, Wauters E et al (2015) Pancreas-specific Sirt1-deficiency in mice compromises beta-cell function without development of hyperglycemia. PLoS One 10:e128012 
41. Fu A, Robitaille K, Faubert B et al (2015) LKB1 couples glucose metabolism to insulin secretion in mice. Diabetologia 58:1513-1522

42. Choe M, Brusgard JL, Chumsri S et al (2015) The RUNX2 transcription factor negatively regulates SIRT6 expression to alter glucose metabolism in breast cancer cells. J Cell Biochem 116:2210 2226

43. Muller MS, Obel LF, Waagepetersen HS, Schousboe A, Bak LK (2013) Complex actions of ionomycin in cultured cerebellar astrocytes affecting both calcium-induced calcium release and storeoperated calcium entry. Neurochem Res 38:1260-1265
44. Uchida K, Tominaga M (2014) The role of TRPM2 in pancreatic beta-cells and the development of diabetes. Cell Calcium 56:332-339

45. Yosida M, Dezaki K, Uchida K et al (2014) Involvement of cAMP/EPAC/TRPM2 activation in glucose- and incretin-induced insulin secretion. Diabetes 63:3394-3403

46. Bauer I, Grozio A, Lasiglie D et al (2012) The NAD+-dependent histone deacetylase SIRT6 promotes cytokine production and migration in pancreatic cancer cells by regulating $\mathrm{Ca} 2+$ responses. J Biol Chem 287:40924-40937 